\title{
Specification of spatial relationships in directed graphs of cell signaling networks
}

\author{
Azi Lipshtat, Susana R. Neves, and Ravi lyengar \\ Department of Pharmacology and Systems Therapeutics Mount Sinai School of Medicine New \\ York, NY 10029
}

\begin{abstract}
Graph theory provides a useful and powerful tool for the analysis of cellular signaling networks. Intracellular components such as cytoplasmic signaling proteins, transcription factors and genes are connected by links, representing various types of chemical interactions that result in functional consequences. However, these graphs lack important information regarding the spatial distribution of cellular components. The ability of two cellular components to interact depends not only on their mutual chemical affinity but also on co-localization to the same subcellular region. Localization of components is often used as a regulatory mechanism to achieve specific effects in response to different receptor signals. Here we describe an approach for incorporating spatial distribution into graphs, and for the development of mixed graphs where links are specified by mutual chemical affinity as well as colocalization. We suggest that such mixed graphs will provide more accurate descriptions of functional cellular networks and their regulatory capabilities and aid in the development of large-scale predictive models of cellular behavior.
\end{abstract}

\section{Keywords}

Signaling networks; intracellular localization; graph theory

The living cell is an excellent example of a dynamically complex system. At any given time there are multiple simultaneously ongoing processes within the cell. Some of these processes such as the production of ATP and other metabolic activities are constitutive while others such as the activity of intracellular signaling pathways are dependent on the presence of certain factors such as extracellular signals. Both types of processes are interconnected and in a healthy cell are balanced with one another. At any given time there are tens to perhaps hundreds of such processes, and they all need to occur in a coordinated manner. How such coordination is achieved and maintained is a central question in biology. A useful approach to understanding large interactive systems is to represent the interacting entities as nodes and the interactions as links in graphs (1). Such graphical representations and their analyses are a well developed area of mathematics called Graph theory (2). In the past decade graph theory has become a very useful tool to analyze various types of networks (3-5). At the intracellular level, these include metabolic and signaling networks. We have used graph theory and network analysis to understand how extracellular signals routed through signaling networks regulate cellular processes (6-8). For these studies, we have used networks where nodes are cellular components and links represent chemical interactions between the components. The definition of links based on mutual chemical specificity of interacting components is a necessary but not sufficient specification for fruitful biological interactions. The components

Address correspondence to: Ravi Iyengar Ph.D., Department of Pharmacology and Systems Therapeutics, Mount Sinai School of Medicine, One Gustave Levy Place Box 1215, New York NY 10029, Voice: 212-659-1707, Fax: 212-831-0114, e-mail ravi.iyengar@mssm.edu. 
also need to be spatially and temporally correlated within the cell. Network representations as static representations do not provide information regarding temporal dynamics, but they should be able to incorporate spatial information. Here we consider how spatial localization can be represented in graphs. We also consider how dual criteria specification of links in graphs representing cellular regulatory networks can be used for better understanding regulatory control processes within cells.

\section{Representation of Cellular Regulatory Networks}

Regulatory networks within cells are often represented as graphs, where nodes correspond to the interacting species such as signaling components and reactants are connected by links to represent direct (or in some cases indirect) chemical interactions. Such graphs can be termed chemical interaction graphs (CIG). This representation simplifies complex systems and enables us to focus on the global view of the system. Many global properties of these networks have been described, including their scale-free topology (3) and small world characteristics (4). In addition, understanding local organizational structures termed network motifs (9) is useful in understanding the regulatory capabilities of these networks (6-8). Thus Graph theory analyses have provided considerable insight into structure/function relationships within complex systems. The performance of a network can be analyzed in increasing levels of details:

\section{Steady state analysis}

Classical Graph theory types of analysis such as connectivity distribution and clustering fall into this category. At this level we ignore the dynamics of the various concentrations of the nodes and the relationships between the levels of nodes and connectivity. We assume that all possible links are engaged and that the system has already converged into a steady state configuration where topology is the main distinguishing characteristic of the network.

\section{Boolean dynamics}

Each node is assigned a Boolean variable, with values of 0 or 1 . These two values correspond to the two possible states of the cellular component represented by the node (high vs. low concentration, active vs. non active, free vs. bound, etc.). The value of each variable is then repeatedly calculated from the values of its neighbors. This is a simple way of simulating the dynamics of a network, and getting a qualitative understanding of the possible contribution of one component (or motif) of the system on the rest of the network.

\section{Quantitative simulation by ODEs}

Quantitative data may be obtained from the network by translating the graph into a set of ordinary differential equations (ODEs). Each node is associated with a number which represents the concentration of the respective component. These concentrations are the variables of the ODEs and change due to the biochemical reactions. This is the most quantitative simulation method. However, in this approach the graph representation is no more than a convenient way of visualization. There is no real use in any of the Graph Theory tools. Such quantitative analyses, although very useful for understanding temporal dynamics often obscure the regulatory topology of networks and do not provide insight into the relationship between different network motifs and the regulatory capabilities that may arise from interactions between network motifs.

In order to comprehend the origins of the processing capabilities of the cell, one must first characterize the dynamic topology of regulatory networks. Once this has been established, more quantitative approaches can be used. Only a method that combines the characterization 
of network motif topology and takes into account the quantitative behavior of these motifs is likely to be able to predict the behavior of complex cellular processes

\section{Spatial Specification of Regulatory Networks}

For most interactions within a cell the chemical ability (i.e. reciprocal affinity) of two components to interact is a necessary but not sufficient criterion for functional interactions. The components must also share the same subcellular location so that they can interact. Spatial distribution plays an important role in regulating and constraining intracellular dynamics. Different localizations of cellular components can promote or prevent certain reactions. Thus, differential localization may be a regulatory mechanism often used by cells to achieve specificity of responses. To develop this line of reasoning we focus on one of the best studied protein kinases in biology, protein kinase A (PKA) that is activated by cAMP binding and regulates a plethora of functions in diverse locations within a neuron. We analyzed small but well understood protein kinase A centered signaling network in neurons.

Fig 1 depicts the spatial segregation of PKA-interacting proteins and respective substrates that is not captured in classical interaction graphs. In figure 1,-several PKA-containing complexes with different subcellular localizations are shown. In each case PKA interacts with a different version of the scaffold protein (A-kinase anchoring protein, AKAP) which in turn binds other signaling components such as protein kinases, phosphatases or channels. Most PKA found in the cell is bound to its scaffold protein AKAP (10). In addition to the tethering of PKA, AKAPs are able to bind to a number of other proteins, such as kinases or phosphatases, creating signaling hubs (11). There have been over 20 AKAP genes identified thus far and through the use of splicing the actual number maybe closer to 50 AKAP-type proteins (10). Each AKAP has a specific targeting domain that gives rise to differing localization within the cell (10), allowing for the spatially distinct allocation of PKA signals along with its respective signaling partners. This spatial segregation of a protein kinase and its substrates constitutes a mechanism that promotes specificity of signaling by restricting the number of possible downstream targets. In the example with PKA, distinct patterns of associations are seen in various subcellular locations (Figure 1). This type of spatial information needs to be accurately captured in network representations.

If one were to ignore spatial specification and only consider chemical interactions, we can construct the classical CIG for protein kinase A interactors shown in Figure 2A. This chemical interaction graph contains three types of interactions: the non-directional scaffolding interaction, the directed arrows (activation), and directed plungers (inhibition). All of the binary interactions in this graph have been experimentally validated and hence we could consider the graph to be a correct representation of the PKA network. This however is not the case. The protein kinase A chemical interaction graph in Fig 2A implies distal relationships that are not correct if one takes into account the spatial segregation of protein kinase A provided by the differential distribution of AKAPs and its binding partners. For example, AKAP450 is localized to the centrosome $(12,13)$ and is able to bind, along with protein kinase A, the phosphatases PP1 and PP2A, and the phosphodiesterase PDE4D3 (14). There has been reports of PKA phosphorylating PDE4D3-and PP1 dephosphorylating it and given their mutual association to AKAP450 this is highly probable. A splice variant of AKAP450 is the AKAP Yotiao (15). Yotiao is localized in neurons to their postsynaptic densities in spines, where it forms a complex with the NMDA receptor (15). This splice variant still retains the PP1 binding site but lacks the ability to bind PDE4D3 (16). Even though AKAP450 and Yotiao share PP1 as a binding partner, due to their different subcelllular locations, it is unlikely that that the two pools of PP1 will have the same local substrates. Hence, the AKAP450 complex should not be connected to the Yotiao complexsince, due to spatial constraints it is highly unlikely that any functional connections between 
these two complexes occur. Thus, NMDA or AMPA receptors and PDE4D3 are unlikely to compete for either PKA or PP1 at a local level and more importantly PDE4DE is not likely to locally regulate PKA control of NMDA receptors or AMPA channels through the degradation of cAMP. Similarly from the graph in Figure 2A we could hypothesize that protein kinase $\mathrm{A}$, by regulating calcium channels $\left(\mathrm{Ca}_{\mathrm{v} 1.2}\right)(17)$, could modulate protein kinase C (PKC) phosphorylation of the AMPA receptor channels (AMPAR) (18). However this is not correct since calcium channels are in the dendritic shaft membranes while AMPA receptor channels that give rise to the excitatory postsynaptic potential are in the post synaptic densities in spines. These two examples illustrate the erroneous inferences regarding connectivity and regulation one can arrive at from graphs that do not include spatial information. The subnetworks, shown in Fig 2B, take into account the spatial specification of components and highlight how AKAPs function as location-specific signaling hubs. From this description we can conclude that whereas the standard CIG representation provides information regarding the possibility of a particular reaction occurring it cannot tell us if the reaction will occur, as co-localization of reactants is a requirement for the reaction to occur. Thus to draw valid functional inferences from graphs of regulatory networks it is necessary to include spatial information.

\section{Incorporating Spatial Specification into Graphs}

Including spatial information into a graph that depicts a regulatory signaling network can be done in one of two approaches. In cases where different compartments can be physically defined (such as organelles such as the nucleus, or a subcellular compartment such as cytoplasm, soma, dendrite, etc.) one may modify the network to include compartment as part of a the name of a node. For example, instead of having a single node to represent proteins such as MAP-Kinase 1, 2, one can have two separate nodes - one for nuclear MAPK and one for cytoplasmic MAPK. The two nodes may be connected by a link, representing the translocation event (19). This approach allows certain reactions to be assigned exclusively to the nuclear MAPK species, such as phosphorylation the nuclear kinase MSK(20), without affecting the cytoplasmic MAPK, which may have its own specific substrates such as cytoplasmic phospholipase- $\mathrm{A}_{2}$ (21). Applying this approach to the PKA network is shown in Figure 2C. Such modified networks can be analyzed using any of the methods described above.

A different approach is to include detailed spatial information in the definition of nodes. This is a natural extension of the ODE method. Instead of associating each node with a time dependent concentration, the system is modeled by a set of partial differential equations (PDEs) and each node represents a time and space dependent concentration. Here there is no need to define compartments, as exact cartesian coordinates are included in the concentration definition, and reactions take place only if all the respective reactants are present simultaneously at the same coordinates. A limitation of this type of representation is the loss of topological information that is essential for the identification of motifs.

Simulation of coupled-PDEs usually requires extensive computational resources and is currently impractical for large-scale systems.

One can use the spatial distribution information to construct a new graph where spatial information is used to specify the links. There are cases where experiments reveal spatial distribution and localization of various cellular components. This data can be processed into a network in the following manner: for each pair of components, calculate the correlation coefficient between their respective spatial distributions. The correlation coefficient between concentrations $\rho_{1}(\vec{r})$ and $\rho_{2}(\vec{r})$ is given by 


$$
C_{1,2}=\frac{\int d A \int \rho_{1} \rho_{2} d A-\int \rho_{1} d A \int \rho_{2} d A}{\sigma_{1} \sigma_{2}}
$$

where $\sigma_{i}=\sqrt{E\left(\rho_{i}^{2}\right)-E^{2}\left(\rho_{i}\right)}$ is the standard deviation and the integration is over the whole space. $C_{1,2}$ is a measure of the overlapping between $\rho_{1}(\vec{r})$ and $\rho_{2}(\vec{r})$ (Figure 3). The spatial correlation $\left(\mathrm{C}_{1,2}\right)$ measures the extent to which distribution of component 1 may provide information and serve as a predictor to distribution of component 2. High correlation between components 1 and 2 implies similar localization of the two components. Areas with high concentration of 1 are expected to have also high concentration of 2, and in locations where 1 is absent, 2 cannot have significant concentration, either. Thus, it is enough to measure one of the components at a certain region in order to get good estimation of the two concentrations at that place (Figure 3A). Low correlation is an indication to independent distributions (Figure 3B). When one of the components (e.g. 1) is localized at a particular region, whereas the other component (2) has a broad distribution, their correlation is low. In such a case, knowing the concentration of 1 at a particular point doesn't increase our knowledge about 2 , and vice versa. It should be noted that such low correlation does not necessarily imply a lack of interaction, since a locally concentrated component may be able to interact with a widely distributed component. $\left(\mathrm{C}_{1,2}\right)$ may get negative values as well. Negative correlation (known also as anti-correlation) is a predictive tool just as the positive correlation. However, in cases of negative correlation, high concentration of 1 at a particular location indicates that 2 is expected to be absent from that region, and low concentration of 1 indicates high concentration of 2 (Figure 3C). Here again, like in the high correlation cases, it should be enough to measure one component in order to gain information about local concentrations of the two components.

Consider the case where we would link any two components whose correlation coefficient is above an user defined threshold (for example 0.8). The resultant graph is the spatial colocalization graph (SCG). In the protein kinase A example shown in Figure 1, the scaffold proteins Yotiao and AKAP450 will not be connected in a SCG although they are closely related in the chemical interaction graph. An SCG can be analyzed using conventional Graph theory metrics to find clusters and pathways which may indicate critical intracellular areas and routes. More importantly, the SCG can be used as a filter for the chemical interaction graph. The two graphs have the same set of nodes (representing intracellular components). In most cases, only pairs of components that are linked in both graphs have fulfilled the requirements for interaction from the biochemical and the spatial criteria. This way the spatial information filters out interactions which are possible biochemically but do not occur in a particular instance due to lack of colocalization between interacting components.

Current experiments as yet do not provide data sets of localization of intracellular components that allows us to construct an intracellular SCG. Thus in the system described in figure 1, we know where individual components are localized, thse are from different studies. So to illustrate the spatial co-localization graph, we have analyzed the data of Petyuk et.al (22). This study describes the spatial distributions of over than 1000 proteins in the brain. It should be emphasized that this study does not include subcellular localization, but rather tissue level distribution. Nevertheless, this is the first study that describes such detailed spatial distribution on a large scale. There are ongoing efforts to conduct high throughput imaging of intracellular proteins (23), but these large-scale datasets are not yet publicly available. From the Petyuk et al study, we downloaded the distributions of all the available proteins, and calculated the correlations between any pair of them. Most of the 
proteins are well localized, indicating their concentrations are non-zero in a defined region of the brain, and zero in the rest of the other regions. About 1/3 of the proteins had positive concentrations throughout the whole brain, indicating they are broadly distributed components. Despite their low correlation with all other components, these proteins can, chemical specificity permitting, interact with any other protein irrespective of the spatial distribution. To examine the effect of the broadly distributed components on the SCG we performed our analysis twice - once with all proteins, including those that have a wide distribution, albeit at varying levels and again with only the localized proteins. The number of protein pairs (i.e. specification of links), whose correlation is greater than a certain threshold is presented as function of the threshold in Figure 4. When considering the whole data set (including the widely distributed proteins), the number of links decreases exponentially with the threshold, until the value of 0.8 . Beyond that point there is a dramatic drop in the number of protein pairs with higher correlation. This sharp change is not seen in the respective plot relating exclusively to localized proteins (dashed plot in Figure 4). This difference indicates that within the subset of widely distributed proteins, the typical correlation is in the range of 0.8-0.95. At the very high correlation range $\left(C_{1,2}>0.9\right)$ the difference between the two plots gets smaller, and they coincide at the end $\left(\mathrm{C}_{1,2}=1\right)$. Interestingly, there are about 20 pairs of proteins that are $99 \%$ correlated, and these proteins are all well localized.

The SCG provides a new tool for understanding cellular regulation. As a threshold has to be determined while constructing the SCG, different threshold values produce different graphs and provide different information respectively. For example, taking a very high threshold would result in the graph only component pairs that are localized together in a very small region such as the $99 \%$ correlation described above. This high correlation indicates tight colocalization and thus indicates either a physical compartment, like nucleus, or common scaffold which is shared by the two correlated components. Even if there is no evidence for mutual chemical affinity between such tightly correlate components the spatial correlation can direct us to look for interactions, both direct and indirect ones, that may be mediated through scaffolds or anchoring components. Spatial correlation can also help to understand the functional role of a known chemical interaction. As mentioned above, correlation can be either positive or negative (Fig. 3). Whereas positive correlation indicates that the two components are co-localized and with appropriate chemical specificity an interaction will occur, negative correlation is an indication of the presence of one component and absence of the other. If the components have the chemical ability to interact with one another, defining a negative threshold and leaving only pairs of components whose correlation is below that threshold, gives us a graph in which each link may predict a regulatory locus, where the movement of a component is used to control chemical interaction and thus achieve local control of a subcellular process. Such regulation can be either direct or indirect. The exact pathway between the negatively correlated components would be found in the chemical interaction data that specifies binary interaction capabilities, but the functionality of such a pathway would be revealed by the differential spatial distribution.

However, considering spatial distribution by itself can result in erroneous representation of the system. This error arises from the fact that some cellular components may be broadly distributed, and nevertheless interacts with locally concentrated components. This situation can be seen by the analyses of the data of Petyuk et al. Using a threshold of 0.8 , yields a graph consisting of 532 nodes (proteins) and about 44000 links (pairs of proteins with higher correlation than the threshold) (Figure 5A). The high density of links in the major island in this graph arises from the high correlation of the widely distributed components. This overwhelming connectivity obscures any meaningful information which may emerge from this graph. If the SCG in Figure 5A is filtered by considering only co-localized proteins then the system is reduced to 224 links between 142 proteins (Figure 5B). However, from visual 
inspection of Figure 5B it can readily be seen that the system is no longer a network but a set of isolated islands. This view is also not correct since it is likely that some of the broadly distributed proteins will interact with some of the local proteins and thus give rise to a better connected network rather that a set of islands. Thus the systems visualized in Figures 5A and $5 \mathrm{~B}$ represent two extremes of the application of the spatial specification criteria and neither are realistic representations. Taking the system in Figure 5A, if we eliminate the links where mutual chemical affinity makes the interactions infeasible then we would obtain a much less densely interactive network. Such analyses is not wholly feasible for the Petyuk et al data since this is tissue not cellular localization, however the framework for mixed graphs where both localization information and mutual chemical affinity are used to specify links are described using a toy system.

\section{Mixed graphs: understanding cellular regulation by analysis of spatial correlation graphs integrated with chemical interaction graphs}

Both chemical specificity and co-localization of the reactants are necessary conditions for a reaction to occur. Thus, it makes sense to construct a multi-layer graph, where two components are connected only if both conditions are fulfilled. However, as demonstrated in Figure 5, the non-localized, widely distributed components require special treatment. These components, chemical specificity permitting, may interact with other components even if their respective correlation is low. Hence, the multilayered graph has to include the widely distributed components with all their chemical specificity links (regardless of spatial correlation), and the localized components whose links are only those links that are present in both the spatial and chemical interaction graphs. A toy example is presented in Figure 6. This example consists of 10 components, of which 5 are widely distributed (blue nodes, numbers 1 through 5) and the other 5 are localized (red nodes 6-10). The SCG consists of all possible links between the non-localized components, in addition to some co-localizations of well localized components (Figure 6A). Drawing the SCG solely for the localized components yields a non-informative graph (Figure 6B). The chemical specificity constraints are given by the CIG (Figure 6C). For constructing the mixed graph we would like to combine the SCG with the CIG in the following way: for the widely distributed components (inner nodes 1-5) we take all of the CIG links. However, between the localized components (nodes 6-10) we consider only links which exist both in the SCG and in the CIG. Thus, for example, links (3 to 9) and (4 to 9) which do not appear at the SCG will be included at the final graph, since they connect non-localized components ( 3 and 4 , respectively). Interaction between components 6 and 7 (or 7 and 10) is possible biochemically, however, since the two reactants are not co-localized, these interactions should be excluded from the multilayered graph (Figure 6D). Similarly, components 8 and 9 are co-localized, but in that case the lack of chemical specificity prevents them from interacting. The resulting mixed graph (Figure 6D) provides an integrative information which is represents a more accurate picture of all the interactions within the system than the CIG or the SCG by themselves. Such a mixed graph can be used for both steady state analysis and dynamical simulations. For dynamic simulations, the correlation coefficient associated with each link can be used as a multiplicative factor altering the overall rate (i.e. concentration of reactants multiplied by the kinetic rates) to yield "effective" reaction rates. This reflects the fact that for any given pair of reactants, only the correlated fraction of each reactant can be involved in the reaction and not the entire pool, which may be located at many other places. This way the spatial information can not only affect the topology of the network but also the dynamics of the various components. 


\section{Perspective}

Recent studies in our laboratory have shown that the dynamics of locally elevated concentrations of signaling components depends on the topology of the interaction network within which these components function (24). Signal transmission through regulatory networks not only involves information regarding the activity state of the component but also information about the location of the active component. This type of study based on partial differential equation is very useful in understanding the spatial dynamics of key signaling component and how spatial information is transmitted from upstream to downstream components with a given pathway or network. However the role of network topology, especially the location of network motifs both in the chemical interaction space as well as subcellular location is not easily deduced from such studies. For this both the CIG and SCG are needed. Hence approaches that allow for facile Graph theory based computation of mixed CIG and SCG will be very useful in understanding and predicting complex cellular regulation

\section{Acknowledgments}

This research was supported by NIH grants GM 072853 and DK-038761 and the Center for Systems Biology

\section{References}

1. Strogatz SH. Exploring complex networks. Nature. 2001; 410:268-276. [PubMed: 11258382]

2. Bollobás, B. Modern Graph Theory. Graduate Texts in Mathematics. Vol. 184. Springer; New york: 1998.

3. Barabási AL, Albert R. Emergence of Scaling in Random Networks. Science. 1999; 286:509-512. [PubMed: 10521342]

4. Watts D, Strogatz S. Collective dynamics of 'small-world' networks. Nature. 1998; 393:440-442. [PubMed: 9623998]

5. Alon U. Biological Networks: The Tinkerer as an Engineer. Science. 2003; 301:1866-1867. [PubMed: 14512615]

6. Ma'ayan A, et al. Formation of regulatory patterns during signal propagation in a mammalian cellular network. Science. 2005; 309:1078-1083. [PubMed: 16099987]

7. Bromberg KD, Ma'ayan A, Neves SR, Iyengar R. Design logic of a cannabinoid receptor signaling network that triggers neurite outgrowth. Science. 2008 in press.

8. Lipshtat A, Purushothaman SP, Iyengar R, Ma'ayan A. Functions of Bifans in Context of Multiple Regulatory Motifs in Signaling Network. Biophys J. 2008; 94:2566-2579. [PubMed: 18178648]

9. Milo R, Shen-Orr S, Itzkovitz S, Kashtan N, Chklovskii D, Alon U. Network Motifs: Simple Building Blocks of Complex Networks. Science. 2002; 298:824-827. [PubMed: 12399590]

10. Michel JJ, Scott JD. AKAP mediated signal transduction. Annu Rev Pharmacol Toxicol. 2002; 42:235-257. [PubMed: 11807172]

11. Smith FD, Langeberg LK, Scott JD. The where's and when's of kinase anchoring. Trends Biochem Sci. 2006; 31:316-323. [PubMed: 16690317]

12. Takahashi M, Shibata H, Shimakawa M, Miyamoto M, Mukai H, Ono Y. Characterization of a novel giant scaffolding protein, CG-NAP, that anchors multiple signaling enzymes to centrosome and the golgi apparatus. J Biol Chem. 1999; 274:17267-17274. [PubMed: 10358086]

13. Witczak O, Skalhegg BS, Keryer G, Bornens M, Tasken K, Jahnsen T, Orstavik S. Cloning and characterization of a cDNA encoding an A-kinase anchoring protein located in the centrosome, AKAP450. Embo J. 1999; 18:1858-1868. [PubMed: 10202149]

14. Tasken KA, Collas P, Kemmner WA, Witczak O, Conti M, Tasken K. Phosphodiesterase 4D and protein kinase a type II constitute a signaling unit in the centrosomal area. J Biol Chem. 2001; 276:21999-22002. [PubMed: 11285255] 
15. Lin JW, Wyszynski M, Madhavan R, Sealock R, Kim JU, Sheng M. Yotiao, a novel protein of neuromuscular junction and brain that interacts with specific splice variants of NMDA receptor subunit NR1. J Neurosci. 1998; 18:2017-2027. [PubMed: 9482789]

16. Westphal RS, Tavalin SJ, Lin JW, Alto NM, Fraser ID, Langeberg LK, Sheng M, Scott JD. Regulation of NMDA receptors by an associated phosphatase-kinase signaling complex. Science. 1999; 285:93-96. [PubMed: 10390370]

17. Hall DD, Davare MA, Shi M, Allen ML, Weisenhaus M, McKnight GS, Hell JW. Critical role of cAMP-dependent protein kinase anchoring to the L-type calcium channel Cav1.2 via A-kinase anchor protein 150 in neurons. Biochemistry. 2007; 46:1635-1646. [PubMed: 17279627]

18. Tan SE, Wenthold RJ, Soderling TR. Phosphorylation of AMPA-type glutamate receptors by calcium/calmodulin-dependent protein kinase II and protein kinase $\mathrm{C}$ in cultured hippocampal neurons. J Neurosci. 1994; 14:1123-1129. [PubMed: 7509863]

19. Chen RH, Sarnecki C, Blenis J. Nuclear localization and regulation of erk- and rsk-encoded protein kinases. Mol Cell Biol. 1992 Mar.12:915-27. 1992. [PubMed: 1545823]

20. Deak M, Clifton AD, Lucocq LM, Alessi DR. Mitogen- and stress-activated protein kinase-1 (MSK1) is directly activated by MAPK and SAPK2/p38, and may mediate activation of CREB. EMBO J. 1998; 17:4426-41. [PubMed: 9687510]

21. Lin LL, Wartmann M, Lin AY, Knopf JL, Seth A, Davis RJ. cPLA2 is phosphorylated and activated by MAP kinase. Cell. 1993; 72:269-78. [PubMed: 8381049]

22. Petyuk VA, Qian WJ, Chin MH, Haixing W, Livesay EA, Monroe ME, Adkins JN, Jaitly N, Anderson DJ, Camp DG II, Smith DJ, Smith RD. Spatial mapping of protein abundances in the mouse brain by voxelation integrated with high-throughput liquid chromatography-mass spectrometry. Genome Res. 2007; 17:328-336. [PubMed: 17255552]

23. Kai H, Murphy R. Boosting accuracy of automated classification of fluorescence microscope images for location proteomics. BMC Bioinformatics. 2004; 5:78. [PubMed: 15207009]

24. Neves SR, Tsokas P, Sarkar A, Grace EA, Rangamani P, Taubenfeld SM, Alberini CM, Schaff JC, Blitzer RD, Moraru II, Iyengar R. Cell shape and negative links in regulatory motifs together control spatial information flow in signaling networks. Cell. 2008 in press. 


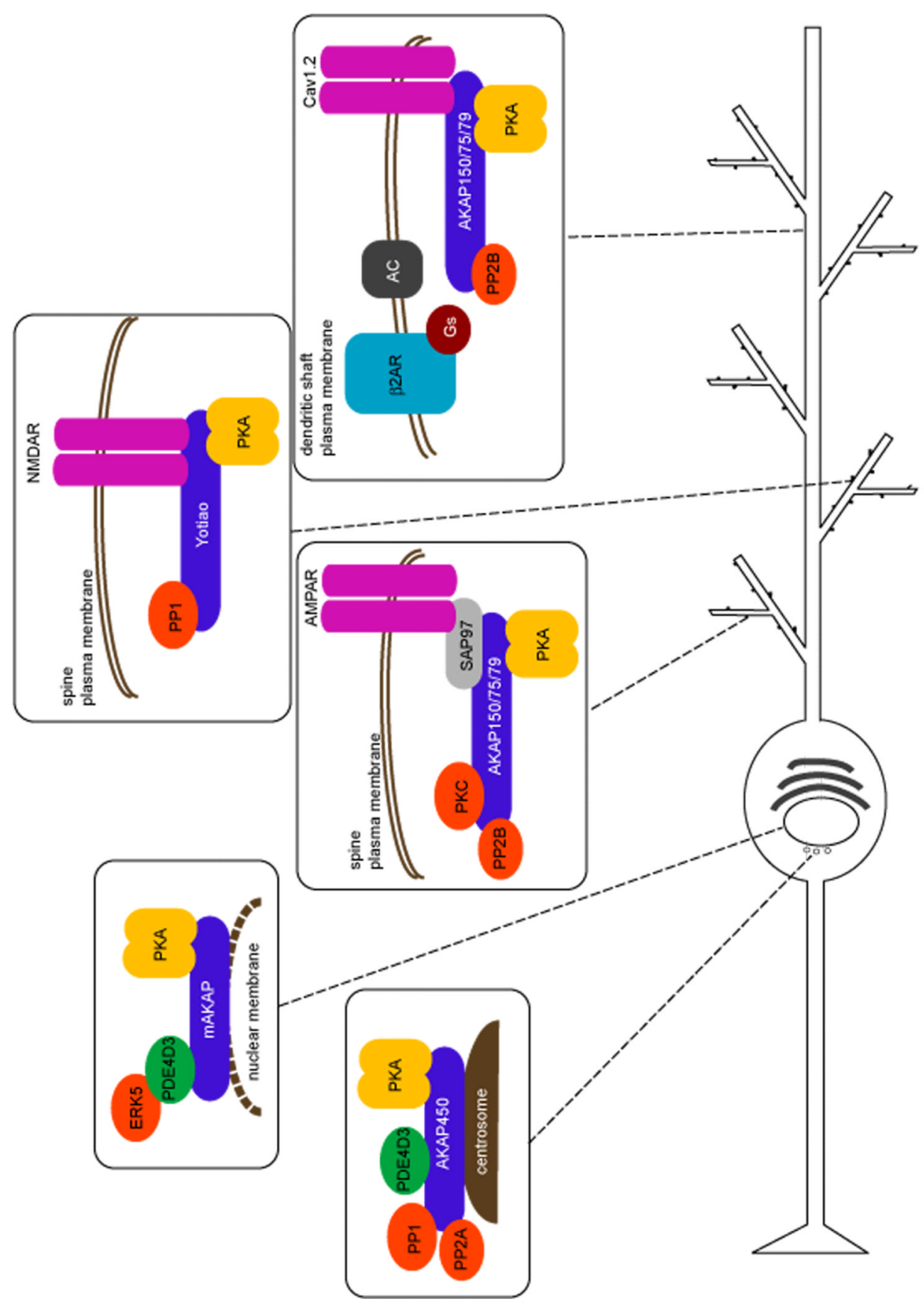

Figure 1.

Schematic representation of the subcellular localization of protein kinase A (PKA) interacting proteins in a neuron. This schematic figure shows how due to presence of different isoforms of the scaffolding protein different combinations of PKA interacting proteins are colocalized in different regions of the neuron. The abbreviations are as follow: kinases- protein kinase A, PKA, extracellular regulated kinase 5, ERK5 and Protein kinase C, PKC; A-kinase anchoring proteins AKAPs- AKAP450, mAKAP, AKAP150/75/79 and Yotiao (AKAP9); Phosphatases- protein phosphatase 1, PP1, protein phosphatase 2A PP2A and protein phosphatase 2B, PP2B; and Phosphodiesterase PDE4D3; ionic channels- alphaamino-3-hydroxy-5-methylisoxazole-4-propionic acid receptor, AMPAR, N-methyl-d- 
aspartate receptor, NMDAR and L-type Calcium channel Cav1.2. Receptor- beta-adrenergic receptor, $\beta 2 \mathrm{AR}$; heterotrimeric G protein Gs; Adenylyl Cyclase AC 

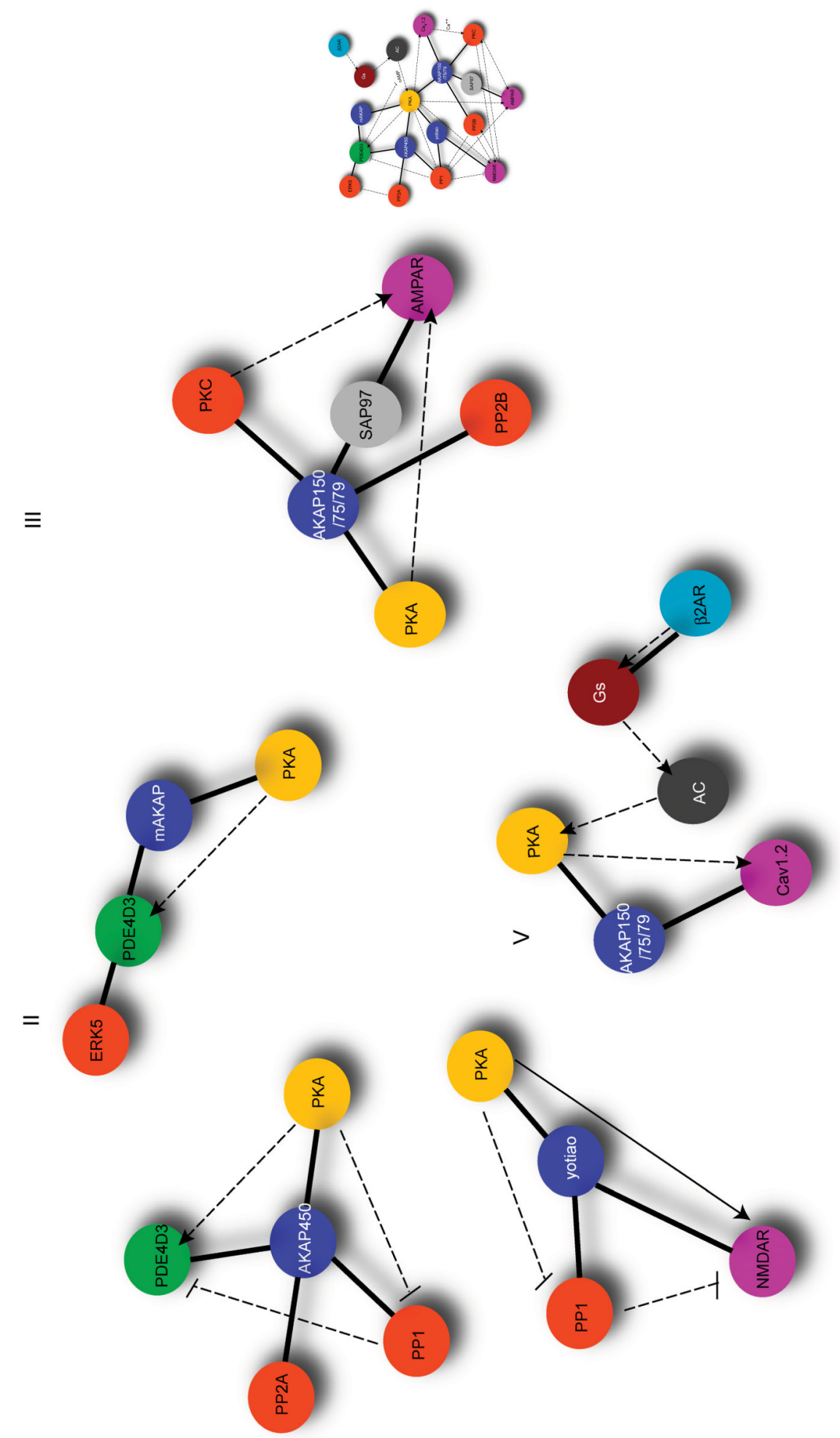


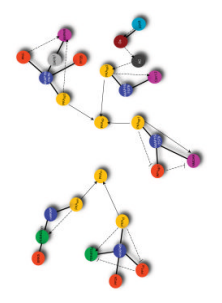

Figure 2.

Protein kinase A interaction networks in neurons A. Protein kinase A-centric protein-protein interaction network without considering localization. Physical interactions are depicted as solid lines. Functional interactions are depicted as dashed arrows. The functional interactions represent enzymatic reactions, conformational changes or non-proteinacious intermediates. B. Subnetworks that result from consideration of spatial localization resulting from localization localization of AKAPs to different regions of the neuron. $\mathbf{C}$. Inclusion of compartment ID as part of the definition of a node. Abbreviations for compartments are as follow: ct centrosome, nm nuclear membrane, n nucleus, sp spine. Please note that there may be a great deal of heterogeneity in the protein composition of each spine. Thus we have sub classified spines into spine 1 , spine 2 and 3 to capture this. 


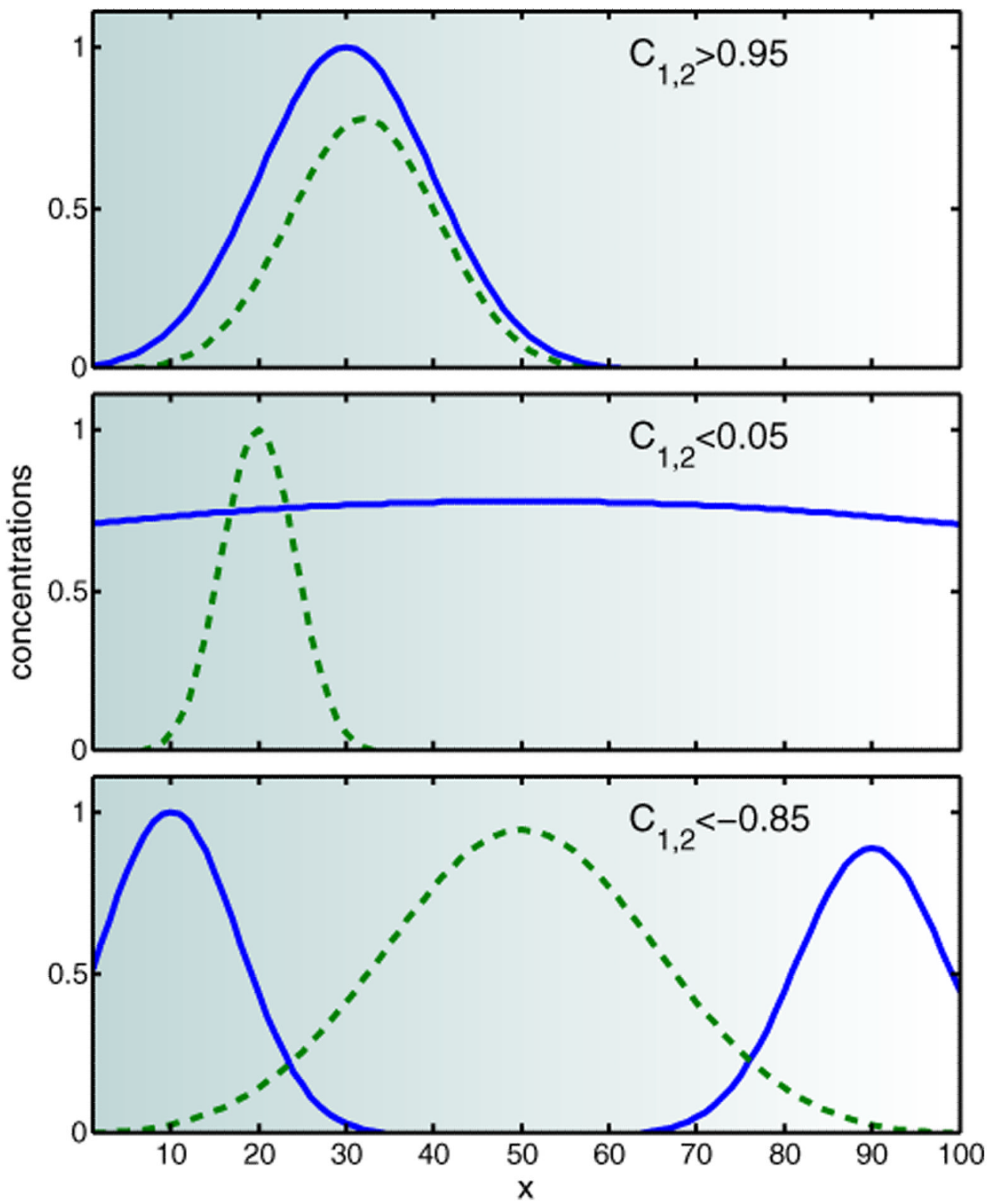

Figure 3.

Demonstration of spatial correlations in a schematic one dimensional cell. Concentrations are normalized to the range from 0 to 1 and space is labeled $\mathrm{x}$ and varies from 0 to 100 . For example this could 1 to 100 microns within a cell. A. High correlation - the two components are localized at the same area. Presence (absence) of one component is a good marker for presence (absence) of the other. B. Low correlation - data regarding one component cannot provide any indication to presence or absence of the other. C. Negative correlation presence (or absence) of one component indicates absence (or presence) of the other. 


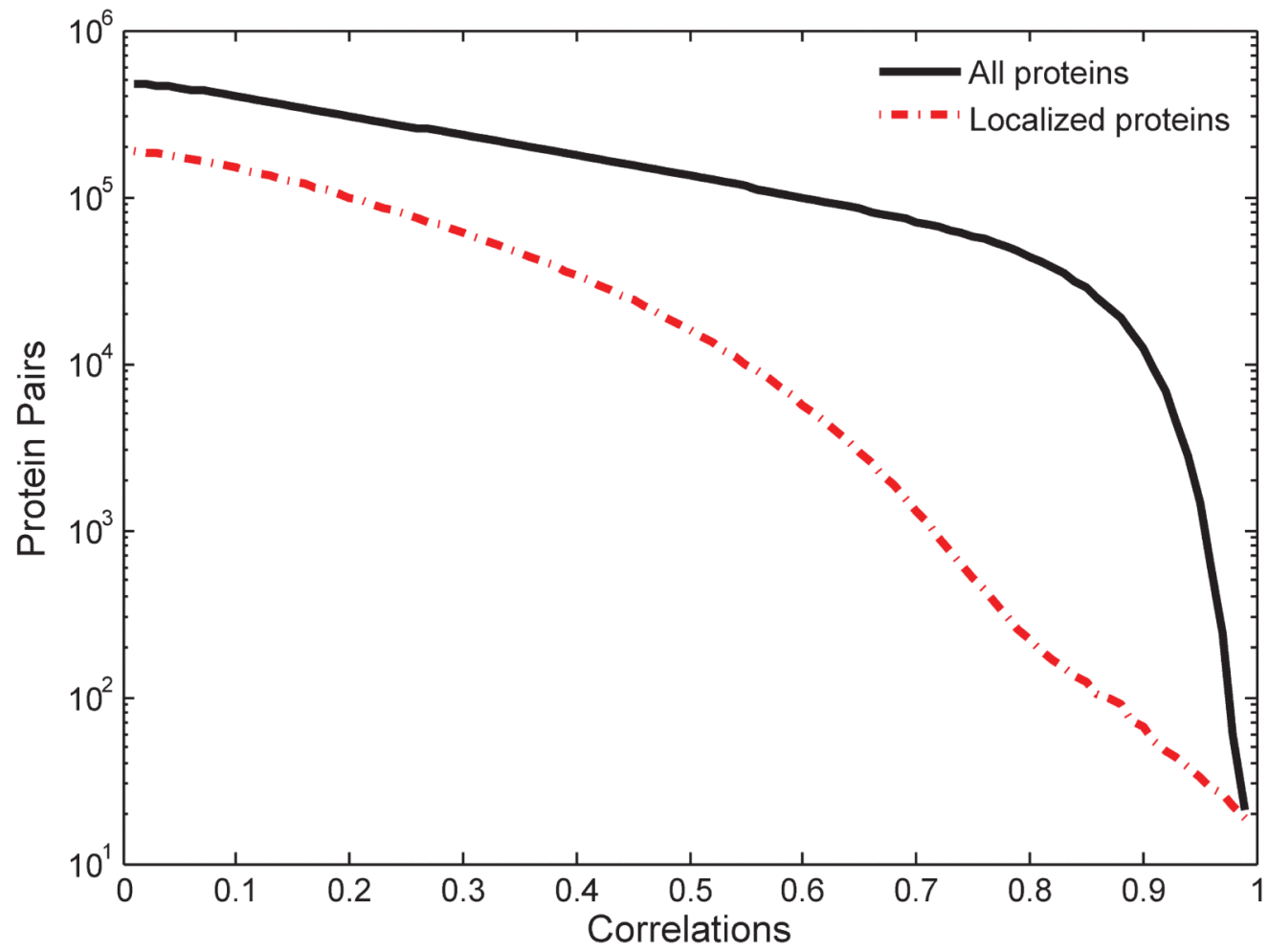

Figure 4.

Number of component pairs whose correlation is greater than a given threshold, as function of the threshold. Localized proteins are defined as proteins with zero concentration at least in one area of the brain as shown by Petyuk et al (22). 20 pairs of proteins have correlation $>0.99$. 


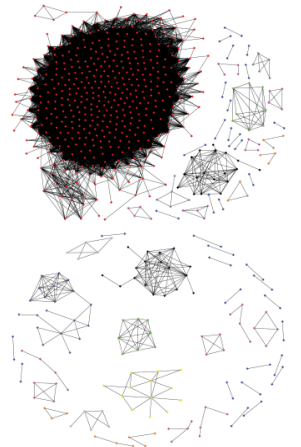

Figure 5.

Spatial correlation graph of the data obtained from Ref. 22, with a threshold 0.8 . The colors indicate size of clusters. A. SCG of the whole data. 532 nodes and $>44000$ links are organized in one large cluster of 433 nodes (in red), one cluster of 18 nodes (black), one cluster of 8 nodes (green) and 2, 5, 3, and 17 clusters of size 5, 4, 3, and 2 respectively (gray, pink, orange, and blue). B. SCG of the same data used in Figure 5A after removing the broadly distributed components. Total of 142 nodes and 224 links in single clusters of sizes 20, 18, 13, 8 each (purple, black, yellow, green, respectively.) and 2, 7, 3, and 18 clusters of size $5,4,3$, and 2 respectively (gray, pink, orange, and blue). 


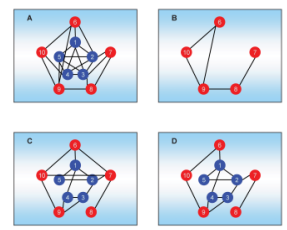

Figure 6.

Demonstration of the steps in building a mixed graph. This toy example consists of 5 widely distributed components (number 1-5) and five localized components (6-10). A. The complete SCG. The widely distributed components are highly connected (similar to the big island in Figure 5A). B. Spatial correlation of the localized components alone (similar to Figure 5B). C. The chemical interaction graph (CIG). D. The mixed graph consists of all the links connected to blue nodes in the CIG (panel C) plus the links which appear both in the SCG and the CIG (intersection of panels B and C). An edge between two nodes in this graph indicates that interaction between the respective two components can occur due to both chemical specificity and spatial co-localization. 